$13 ; 15$

\title{
Микроволновый полосно-пропускающий фильтр на диэлектрических слоях с металлическими сетками
}

\author{
(ㄷ) Б.А. Беляев ${ }^{1,2}$, В.В. Тюрнев ${ }^{1,2}$, А.С. Волошин ${ }^{2,3}$, Р.Г. Галеев ${ }^{3,4}$ \\ ${ }^{1}$ Институт фозики им. Л.В. Киренского ФИЦ КНЦ СО РАН, \\ Красноярск, Россия \\ ${ }^{2}$ Сибирский федеральный университет, Красноярск, Россия \\ ${ }^{3}$ Сибирский государственный университет науки и технологий \\ им. акад. М.Ф. Решетнева, Красноярск, Россия \\ ${ }^{4}$ НПП „Радиосвязь“, Красноярск, Россия \\ E-mail: belyaev@iph.krasn.ru
}

Поступило в Редакцию 31 октября 2017 г.

Разработана конструкция полосно-пропускающего фильтра, состоящего из диэлектрических слоев с металлическими сетками на поверхностях. Диэлектрические слои являются полуволновыми резонаторами, сетки служат зеркалами с заданными отражательными свойствами, обеспечивающими оптимальные связи между резонаторами и оптимальные связи крайних резонаторов со свободным пространством. Изготовленный опытный образец синтезированного фильтра третьего порядка с центральной частотой полосы пропускания $\sim 12 \mathrm{GHz}$ и относительной ее шириной $\sim 17 \%$ показал хорошее согласие теории и эксперимента. Конструкция может использоваться в качестве радиопрозрачных в заданной полосе частот панелей для укрытия микроволновых антенн.

DOI: 10.21883/PJTF.2018.10.46093.17102

В настоящее время исследователи активно изучают особенности распространения электромагнитных волн, падающих на слоистые кон- 
струкции из диэлектрических пластин, на границах раздела которых сформированы периодические структуры из полосковых проводников (2D-решетки или сетки). Интерес к таким конструкциям обусловлен возможностью создания на их основе частотно-селективных поверхностей, служащих полосно-пропускающими фильтрами в диапазонах от субмикронных до дециметровых длин волн [1-4]. Полосковые элементарные ячейки, из которых состоит периодическая 2D-структура, например металлические квадраты или ячейки металлической сетки, являются резонаторами [5]. Именно поэтому, используя многослойные конструкции из таких структур, можно создавать различные полосно-пропускающие фильтры [5,6]. Важно отметить, что длина волны на центральной частоте полосы пропускания в таких фильтрах, которые нередко называют частотно-селективными поверхностями [7], не только много больше периода решеток, но и много больше толщины диэлектрических слоев.

Известно, что сопротивление проводников на высоких частотах растет из-за уменьшения толщины скин-слоя, что приводит с учетом шероховатости подложки к снижению собственной добротности полосковых резонаторов. Поэтому главным недостатком многослойных фильтров, использующих резонансные структуры из полосковых проводников, являются сравнительно большие потери мощности в полосе пропускания устройств. Более перспективными исследователи считают конструкции фильтров, в которых сами диэлектрические слои являются высокодобротными резонаторами, а 1D- или 2D-структуры из полосковых проводников на их поверхностях служат зеркалами с заданной отражательной способностью $[8,9]$. При этом период полосковых структур выбирается значительно меньше длины волны, чтобы их резонансные частоты находились как можно выше полосы пропускания фильтра. В работах $[8,10]$ для таких конструкций получены формулы синтеза фильтров с заданной шириной полосы пропускания и заданным уровнем потерь на отражение в ней, которые позволяют определить оптимальные размеры полосковых проводников на поверхностях каждого из диэлектрических слоев.

В настоящей работе исследована разработанная конструкция полосно-пропускающего фильтра третьего порядка на диэлектрических слоях-резонаторах, разделенных металлическими сетками. Приведено сравнение расчетных и измеренных амплитудно-частотных характеристик (АЧХ) опытного образца фильтра.

Письма в ЖТФ, 2018, том 44, вып. 10 


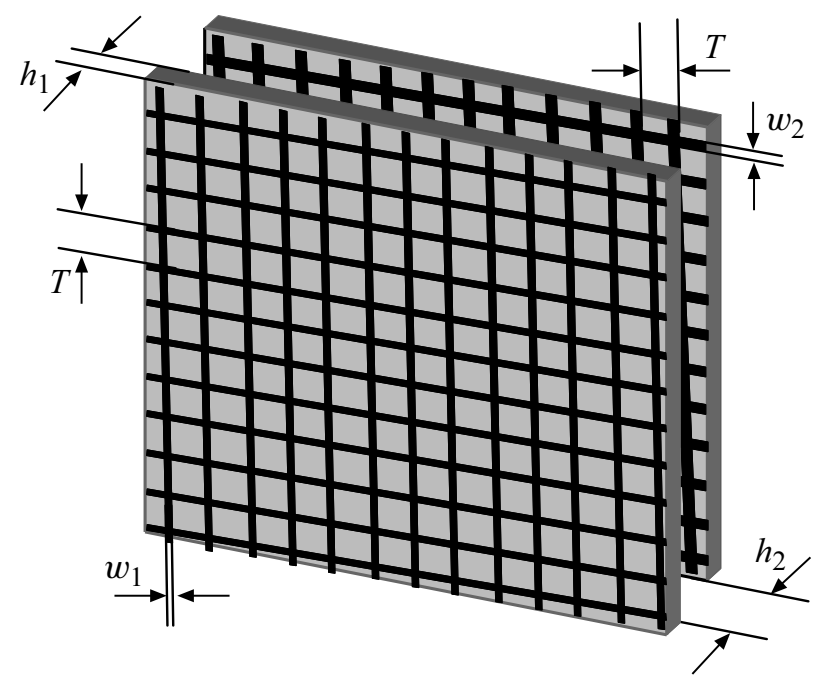

Pис. 1. Конструкция полосно-пропускающего фильтра третьего порядка на диэлектрических слоях с металлическими сетками.

Конструкция исследуемого фильтра содержит две одинаковые параллельные диэлектрические пластины с относительной диэлектрической проницаемостью $\varepsilon_{1}$ и толщиной $h_{1}$, разделенные диэлектрическим слоем, например воздухом, толщиной $h_{2}$ с диэлектрической проницаемостью $\varepsilon_{2}$ (рис. 1). На поверхностях диэлектрических пластин квадратные сетки из металлических проводников имеют одинаковый период $T$. При этом ширина полосковых проводников у наружных сеток равна $w_{1}$, а у внутренних $-w_{2}$. Очевидно, что в рассматриваемой конструкции каждая из диэлектрических пластин и воздушный зазор между ними являются взаимодействующими полуволновыми резонаторами, которые формируют полосу пропускания устройства.

При настройке фильтра необходимо учитывать, что центральная частота его полосы пропускания определяется толщиной диэлектрических слоев, а ширина полосы пропускания и заданный максимальный уровень отражений в ней - подбором ширины полосковых проводников у внутренних и наружных сеток. С увеличением $w_{2}$ и $w_{1}$ связь между соседними резонаторами и крайних резонаторов со свободным про-

Письма в ЖТФ, 2018, том 44, вып. 10 
странством уменьшается, что приводит к уменьшению ширины полосы пропускания фильтра, и наоборот. Заметим, что металлические сетки понижают резонансные частоты резонаторов, причем тем сильнее, чем больше связь между ними.

Наиболее просто расчет АЧХ рассматриваемого фильтра при нормальном падении электромагнитной волны может быть выполнен в квазистатическом приближении матричным методом. Для всего фильтра $A B C D$-матрица (классическая матрица передачи) может быть рассчитана как произведение $A B C D$-матриц каждого компонента конструкции $[11]$, т.е. каждого слоя и каждой сетки. Для определенности будем предполагать, что временна́я зависимость компонент электромагнитного поля описывается множителем $\exp (-i \omega t)$. В этом случае нормированная $A B C D$-матрица для каждого диэлектрического слоя может быть рассчитана по формуле [11]

$$
\left(\begin{array}{ll}
A_{1,2}^{d} & B_{1,2}^{d} \\
C_{1,2}^{d} & D_{1,2}^{d}
\end{array}\right)=\left(\begin{array}{cc}
\cos \theta_{1,2} & -i \frac{\sin \theta_{1,2}}{n_{1,2}} \\
-i n_{1,2} \sin \theta_{1,2} & \cos \theta_{1,2}
\end{array}\right),
$$

где $n_{1,2}=\sqrt{\varepsilon_{1,2}}-$ показатели преломления слоев, а $\theta_{1,2}=\frac{\omega}{c} n_{1,2} h_{1,2}-$ фазовые толщины слоев. Здесь верхний индекс $d$ указывает на то, что $A B C D$-матрица относится к слою диэлектрика.

Матрица рассеяния бесконечно тонкой идеально проводящей металлической сетки, расположенной на границе сред с показателями $n_{1}$ и $n_{2}$, выражается формулами [12]

$$
\begin{aligned}
&\left(\begin{array}{ll}
S_{11}^{m} & S_{12}^{m} \\
S_{21}^{m} & S_{22}^{m}
\end{array}\right)=\left(\begin{array}{ll}
\frac{n_{1}-n_{2}-i Z_{0} Y_{1,2}}{n_{2}+n_{1}+i Z_{0} Y_{1,2}} & \frac{2 \sqrt{n_{2} n_{1}}}{n_{2}+n_{1}+i Z_{0} Y_{1,2}} \\
\frac{2 \sqrt{n_{2} n_{1}}}{n_{2}+n_{1}+i Z_{0} Y_{1,2}} & \frac{n_{2}-n_{1}-i Z_{0} Y_{1,2}}{n_{2}+n_{1}+i Z_{0} Y_{1,2}}
\end{array}\right), \\
& Y_{1,2}= \frac{2 \pi}{\omega \mu_{0}\left(T-w_{1,2}\right) \ln \left(\operatorname{cosec}\left(\frac{\pi w_{1,2}}{2 T}\right)\right)} \\
&-\omega \varepsilon_{0} T \frac{n_{1}^{2}+n_{2}^{2}}{\pi} \ln \left(\sec \left(\frac{\pi w_{1,2}}{2 T}\right)\right),
\end{aligned}
$$

полученными в квазистатическом приближении, где индекс $m$ указывает на металл, а $Z_{0}, \mu_{0}$ и $\varepsilon_{0}-$ характеристическое сопротивление, абсолютная магнитная и абсолютная диэлектрическая проницаемости

Письма в ЖТФ, 2018, том 44, вып. 10 
свободного пространства соответственно. Отсюда элементы нормированной $A B C D$-матрицы металлической сетки могут быть вычислены по формулам [11]

$$
\begin{gathered}
A^{m}=\left(1+S_{11}^{m}-S_{22}^{m}-\operatorname{det}\left[S_{i k}^{m}\right]\right) n_{2} /\left(2 S_{21}^{m} n_{1}\right), \\
B^{m}=\left(1+S_{11}^{m}+S_{22}^{m}+\operatorname{det}\left[S_{i k}^{m}\right]\right) n_{2} /\left(2 S_{21}^{m} n_{1} n_{2}\right), \\
C^{m}=\left(1-S_{11}^{m}-S_{22}^{m}+\operatorname{det}\left[S_{i k}^{m}\right]\right) n_{1} n_{2} /\left(2 S_{21}\right), \\
D^{m}=\left(1-S_{11}^{m}+S_{22}^{m}-\operatorname{det}\left[S_{i k}^{m}\right]\right) /\left(2 S_{21}^{m} n_{1} n_{2}\right),
\end{gathered}
$$

Перемножив $A B C D$-матрицы каждого компонента структуры, можно вычислить матричные элементы $A, B, C$ и $D$ матрицы передачи всей конструкции рассматриваемого фильтра.

Селективные свойства любого фильтра характеризуют частотной зависимостью матричных элементов его матрицы рассеяния S. Коэффициентами отражения являются матричные элементы $S_{11}$ и $S_{22}$, а коэффициентами прохождения - элементы $S_{21}$ и $S_{12}$. Рассматриваемая конструкция является симметричным и взаимным четырехполюсником [11]. Поэтому выполняются равенства $\left|S_{11}\right|=\left|S_{22}\right|, S_{21}=S_{12}$. Вычислив элементы $A B C D$-матрицы фильтра, значения коэффициентов отражения и прохождения несложно получить по формулам [11]

$$
S_{11}=\frac{A+B-C-D}{A+B+C+D}, \quad S_{21}=\frac{2}{A+B+C+D} .
$$

Здесь предполагается, что фильтр окружает среда с показателем преломления $n=1$.

На основе полученных формул была написана компьютерная программа численного анализа рассмотренной конструкции фильтра третьего порядка (рис. 1). С помощью этой программы был проведен параметрический синтез конструктивных параметров фильтра, в котором диэлектрические слои и сетки на их поверхностях изготовлены из металлизированных пластин ТММ3 корпорации Rogers, имеющих толщину $h_{1}=5.08 \mathrm{~mm}$, относительную диэлектрическую проницаемость $\varepsilon_{1}=3.41$ и толщину слоев из меди $17.5 \mu \mathrm{m}$. Заметим, что частота полуволнового резонанса $\left(\lambda_{1} / 2=h_{1}\right)$ в таком диэлектрическом слое равна $f_{1} \approx 15.96 \mathrm{GHz}$. Однако из-за присутствия сеток в фильтре центральная частота его полосы пропускания $f_{0}$, очевидно, будет ниже частоты $f_{1}$.

Письма в ЖТФ, 2018, том 44, вып. 10 


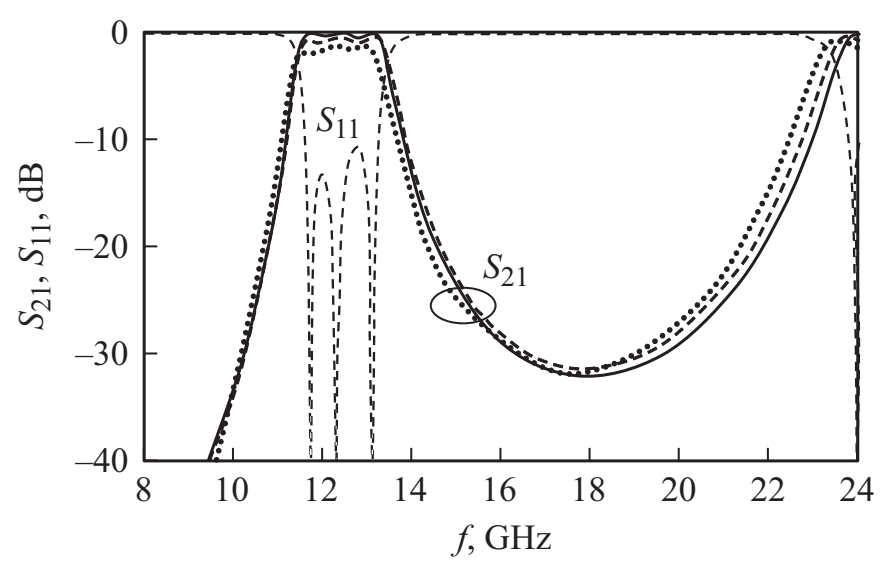

Рис. 2. АЧХ фильтра третьего порядка на диэлектрических слоях с металлическими сетками. Сплошная линия - квазистатический расчет, штриховая линия - электродинамический анализ 3D-модели, пунктир - эксперимент.

Учитывая тот факт, что период сеток должен быть меньше $\lambda_{1} / 2$ [12], в исследуемом фильтре зафиксируем $T=3 \mathrm{~mm}$. Пусть для определенности максимумы отражений в полосе пропускания не превышают $-14 \mathrm{~dB}$. Очевидно, оптимальным величинам соответствующих связей резонаторов друг с другом и со свободным пространством будут отвечать определенные значения $w_{1}$ и $w_{2}$. Для простоты анализа исследуемой конструкции зафиксируем в ней ширину полосковых проводников наружных сеток $w_{1}=0.1 \mathrm{~mm}$.

Таким образом, учитывая все перечисленные выше условия, при параметрическом синтезе фильтра потребуется подобрать лишь два параметра конструкции: ширину полосковых проводников внутренних сеток и толщину воздушного зазора между диэлектрическими пластинами. По результатам синтеза эти параметры принимают соответственно следующие значения: $w_{2}=0.58 \mathrm{~mm}$ и $h_{2}=11.15 \mathrm{~mm}$. АЧХ прямых потерь $S_{21}(f)$ синтезированного фильтра показана сплошной линией на рис. 2. Фильтр имеет центральную частоту полосы пропускания $f_{0}=12.44 \mathrm{GHz}$ и относительную ширину полосы пропускания, измеренную по уровню $-3 \mathrm{~dB}$ от уровня минимальных потерь, $\Delta f / f_{0}=16.7 \%$. 


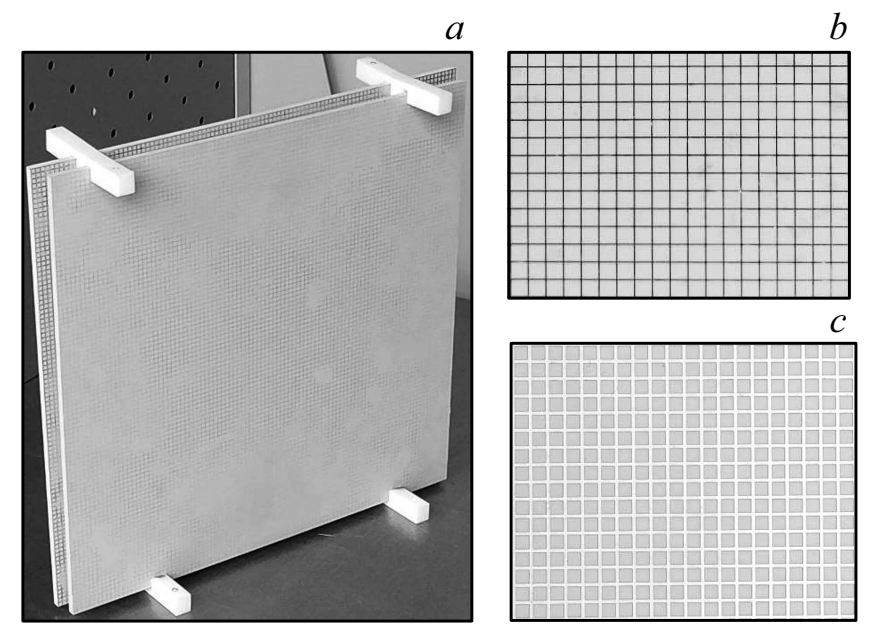

Рис. 3. Фотография опытного образца фильтра третьего порядка на диэлектрических слоях с металлическими сетками $(a)$ и увеличенные фрагменты его наружных $(b)$ и внутренних $(c)$ сеток.

Для проверки точности полученных формул нами в электродинамическом пакете CST Microwave Studio были рассчитаны амплитудно-частотные характеристики 3D-модели фильтра, показанные штриховыми линиями на рис. 2. Учет омических потерь в проводниках сеток увеличил минимальные потери в полосе пропускания фильтра от 0 до $0.32 \mathrm{~dB}$, однако зависимости $S_{21}(f)$ и $S_{11}(f)$ при этом почти не изменились. Центральная частота полосы пропускания фильтра $f_{0}=12.41 \mathrm{GHz}$, а относительная ширина полосы пропускания, измеренная по уровню $-3 \mathrm{~dB}$ от уровня минимальных потерь, $\Delta f / f_{0}=16.8 \%$.

Экспериментальная проверка расчетов была проведена на опытном образце фильтра, изготовленном из диэлектрических пластин TMM3 корпорации Rogers, имеющих толщину $h_{1}=5.08 \mathrm{~mm}$ и размеры $300 \times 300 \mathrm{~mm}$. Фотография слоистого фильтра представлена на рис. $3, a$, а увеличенные фрагменты его наружных и внутренних сеток показаны на рис. $3, b$ и $c$ соответственно. После изготовления медные сетки были покрыты серебром для защиты от окисления. В фильтре пластины скреплялись параллельно друг другу на расстоянии $h_{2}=11.15 \mathrm{~mm}$ дву-

Письма в ЖТФ, 2018, том 44, вып. 10 
мя парами небольших тефлоновых держателей. АЧХ опытного образца фильтра, представленная пунктиром на рис. 2, регистрировалась на векторном анализаторе цепей ZVK фирмы Rohde \& Schwarz (Германия) c помощью широкополосных измерительных антенн. Относительная ширина полосы пропускания экспериментального фильтра, измеренная по уровню $-3 \mathrm{~dB}$ от уровня минимальных потерь, $\Delta f / f_{0}=16.5 \%$, ее центральная частота $f_{0}=12.29 \mathrm{GHz}$, а минимальные потери в полосе пропускания $1.15 \mathrm{~dB}$.

Таким образом, предложенная конструкция, состоящая из диэлектрических слоев, разделенных металлическими сетками, может служить полосно-пропускающим фильтром. Важно отметить, что слои могут изготавливаться как из одного, так и из разных материалов. Очевидно, что из панелей, изготовленных на основе таких слоистых структур, можно конструировать радиопрозрачные поверхности для защиты параболических антенн и антенных решеток [13].

Работа выполнена при поддержке Министерства образования и науки РФ (соглашение № 14.575.21.0142, уникальный идентификатор проекта RFMEFI57517X0142).

\section{Список литературы}

[1] Melo A.M., Kornberg M.A., Kaufman P., Piazzettaet M.H., Bortoluccial E.C., Zakia M.B., Bauer O.H., Poglitsch A., Alves da Silva A.M.P. // Appl. Opt. 2008. V. 47. N 32. P. 6064-6069.

[2] Garcia-Vidal F.J., Martin-Moreno L., Ebbesen T.W., Kuipers L. // Rev. Mod. Phys. 2010. V. 82. N 1. P. 729-788.

[3] Tomasek P. // Int. J. Circ. Syst. Signal Proc. 2014. V. 8. P. 594-599.

[4] Oh S., Lee H., Jung J.-H., Lee G.-Y. // Int. J. Microwave Sci. Technol. 2014. V. 2014. P. $857582(1-5)$.

[5] Ade P.A.R., Pisano G., Tucker C., Weaver S. // Proc. SPIE. 2006. V. 6275. P. $62750 \mathrm{U}-1$.

[6] Zhou H., Qu S.-B., Wang J.-F., Lin B.-Q., Ma H., Xu Z., Bai P., Peng W.-D. // Electron. Lett. 2012. V. 48. N 1. P. 11-12.

[7] Munk B.A. Frequency selective surfaces: theory and design. N.Y.: WileyInterscience, $2000.410 \mathrm{p}$.

[8] Belyaev B.A., Tyurnev V.V. // Opt. Lett. 2015. V. 40. N 18. P. 4333-4335.

[9] Abadi S.M.A.M.H., Behdad N. // IEEE Transact. Antennas Propagation. 2015. V. 63. N 11. P. 4766-4774. 
[10] Belyaev B.A., Tyurnev V.V. // Opt. Lett. 2016. V. 41. N 3. P. 536-539.

[11] Гупта К., Гардж Р., Чадха Р. Машинное проектирование СВЧ устройств. М.: Радио и связь, 1987. 104 с.

[12] Беляев Б.А., Тюрнев В.В. // РЭ. 2017. Т. 62. № 7. С. 642-650.

[13] Mainwaring А., Умнов А.Л., Шуралев М.О., Ельцов А.Ю. // Письма в ЖТФ. 2011. T. 37. B. 4. C. $68-75$.

Письма в ЖТФ, 2018, том 44, вып. 10 